\title{
Nicodemo, un camino de discipulado
}

\author{
Nicodemus, a path of discipleship
}

Jaime Alberto Pérez Villegas*

* Mestre pelo Pontifício Insti-
tuto Bíblico. Diretor de Car-
reira da Licenciatura em Ci-
ências Religiosas da Universi-
dade Católica de Manizales,
Manizales, Colombia.
jaipervi@hotmail.com

Recebido em: 26/10/2021

Aprovado em: 19/12/2021

Licença Creative Commons CC BY 4.0 (c) (i)

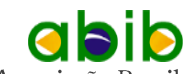

Associação Brasileira de Pesquisa Bíblica

\section{Resumen}

El Evangelio de Juan permite sumergirse a través del personaje de Nicodemo en la aventura del creer, de la escucha, de la acción y del reconocimiento. Aparece en tres momentos sumamente importantes para el evangelista: al inicio del ministerio de Jesús en Jerusalén; luego, en la fiesta de las Tiendas, cuando llega la división entre quienes creen en Él y quienes no y, por último, en el momento de su muerte. En cada uno de ellos se presentan elementos que dejan ver los diferentes modos de responder a la persona de Jesús: de modo superficial, en contra de Él, o con una adhesión comprometida en acciones concretas. Así, Nicodemo sirve al narrador para presentar un proceso de discipulado, con cambios progresivos tanto en la percepción como en la acción; por eso, la trama narrativa del personaje permite ver la evolución de su fe, sus ambigüedades, su timidez y, finalmente, sus decisiones.

Palabras clave: Discipulado. Proceso. Maestro. Camino. Narrativa.

\begin{abstract}
The Gospel of John allows one to immerse oneself through the character of Nicodemus in the adventure of believing, listening, acting and recognizing. He appears at three extremely important moments for the evangelist: at the beginning of Jesus' ministry in Jerusalem; then, at the Feast of the Tents, when it comes the division between those who believe in Him and those who do not and, finally, at the moment of his death. In each of them there are elements that reveal the different ways of responding to the person of Jesus: superficially, against Him, or with a committed adherence to concrete actions. Thus, Nicodemus serves the narrator to present a process of discipleship, with progressive changes in both perception and action; For this reason, the narrative plot of the character allows us to see the evolution of his faith, his ambiguities, his shyness and, finally, his decisions.
\end{abstract}

Keywords: Discipleship. Process. Master. Path. Narrative.

\section{Resumo}

O Evangelho de João permite submergir através do personagem Nicodemos na aventura do crer, da escuta, da ação e do reconhecimento. Ele aparece em três momentos sumamente importantes para o evangelista: no início do ministério de Jesus em Jerusalém; 
então, na festa das Tendas, quando chega a divisão entre os que creem nEle e os que não e, por último, no momento da sua morte. Em cada um deles, apresentam-se elementos que permitem ver os diferentes modos de responder à pessoa de Jesus: de modo superficial, contra Ele, ou com uma adesão comprometida em ações concretas. Assim, Nicodemos serve ao narrador para apresentar um processo de discipulado, com as mudanças progressivas tanto na percepção quanto na ação; por isso, a trama narrativa do personagem permite ver a evolução de sua fé, suas ambiguidades, sua timidez e, finalmente, suas decisões.

Keywords: Discipulado. Processo. Mestre. Caminho. Narrativa.

\section{Introducción}

El personaje de Nicodemo en la Sagrada Escritura sólo se encuentra en el Evangelio de Juan, de allí que un acercamiento a su personalidad debe ser abordado desde la perspectiva joánica, donde la fe y la respuesta a la propuesta de Jesús ocupan un lugar central; su análisis nos permitirá, entonces, identificar un camino de discipulado, el cual se vive de manera progresiva, un verdadero proceso que supone cambios graduales tanto en la percepción de lo que significa ser discípulo como en el compromiso concreto que esto conlleva.

El nombre de Nicodemo se encuentra cinco veces en el Evangelio de Juan en tres textos distintos a lo largo de todo el escrito, su presencia allí no es de tipo accidental, por el contrario, es fácil constatar que, aunque en un inicio se presenta de un modo misterioso, progresivamente cobra protagonismo hasta aparecer en uno de los momentos culmen de la narración joánica.

Acercarse a los personajes del Evangelio de Juan, exige estar atento a los detalles, recordando que cada uno tiene una misión propia en la narración, por tal motivo, es indispensable considerar a Nicodemo en todo su conjunto; no es posible emitir un juicio sobre él, si se accede a los textos de modo parcial, de hecho, la visión general dará elementos sólidos para profundizar en un hombre lleno de ambiguiedades, pero al final también de decisiones.

La trama narrativa ${ }^{1}$ de Nicodemo nos permite ver su proceso de fe, sus ambigüedades, su timidez, pero, sobre todo, nos permite descubrir su presencia, que se vuelve provocadora al lector; se invita constantemente a tomar decisiones, y a buscar las verdaderas razones del por qué vale la pena seguir a Jesús.

La valoración sobre Nicodemo ha sido bastante discutida y para muchos autores su presencia en el Evangelio permanece ambigua desde el inicio hasta el final; para otros, la valoración es negativa, pues el personaje no se representa a sí mismo, sino más bien tiene un carácter colectivo que personifica a una clase de la sociedad judía resistente a aceptar a Jesús; por último, un numeroso grupo de autores piensa que Nicodemo, en medio de las dificultades, se puede considerar como un verdadero discípulo. La línea de reflexión del presente artículo presenta una valoración positiva, por las razones que se indicarán: Nicodemo debe confrontar su vida y su opción por Jesús y desde allí tomar una decisión, la cual se muestra al final del Evangelio en el signo de la sepultura de quien es ya su Maestro y su Rey.

\footnotetext{
${ }^{1}$ Analizada a la luz de Marguerat y Bourquin (2011), Zapella (2014) y Culpepper (1983).
} 


\section{El personaje en su contexto}

Como se ha mencionado, el nombre de Nicodemo aparece en tres textos, en tres lugares distintos a lo largo del Evangelio de Juan, los detalles de cada uno de ellos permiten una aproximación cercana a reconocer quién es realmente este personaje. Un primer detalle interesante es que está presente en tres momentos sumamente importantes para Juan: al inicio del ministerio de Jesús en Jerusalén; después, en la fiesta de las Tiendas, cuando llega la división entre quienes creen en Él y quienes no; por último, en el momento de su muerte.

Podríamos clasificar en tres categorías el modo como Nicodemo es presentado:

- Presentación Genérica: En el capítulo 3 se dice que es un hombre, ya en el capítulo 2,23-25, se menciona que un grupo de hombres creían en Jesús, pero Él no se fiaba de ellos; Nicodemo era de este tipo de hombres. Igualmente, se señala a Nicodemo como fariseo; entendiendo los fariseos como aquellos que se consideraban los legítimos representantes del judaísmo postexílico; para ellos el verdadero Israel estaba formado únicamente por quienes observan minuciosamente la Torá, es decir, ellos eran el único verdadero Israel.

- Presentación Personal: Se menciona su nombre Nicodemo ${ }^{2}$. Jefe de los judíos, miembro del Sanedrín ${ }^{3}$ seguramente, (por tanto, un verdadero un judío).

- Presentación Cognitiva: En el capítulo 3, el narrador lo presenta como aquel que tiene conocimiento. Según Jesús, es maestro de Israel. Sin embargo, el mismo Jesús lo confronta como el que no sabe estas cosas, por tanto, se ve el contraste entre el que sabe, el maestro y el ignorante; por último, el narrador da a entender que es un hombre silencioso.

El primer texto, sin duda, ofrece una trama de revelación progresiva, es amplio el diálogo entre los dos personajes principales: Jesús y Nicodemo, lo que permite conocer, desde un inicio, el planteamiento teológico que el autor quiere presentarnos a la luz de la figura de Nicodemo. El segundo relato presenta una segunda complicación y en el último texto, de un modo casi solemne y real, la solución a las complicaciones anteriores por fin aparece. Podemos concluir que el telling que nos da el narrador sobre Nicodemo es amplio y detallado, la descripción de tipo genérica y personal dan testimonio de ello. El showing, se encuentra en su presentación cognitiva y en descubrir que es un hombre en movimiento, rico y generoso, pues sus acciones así lo demuestran.

A continuación se profundizará en las tres menciones que hace el evangelista Juan acerca de Nicodemo; esto nos dejará ver los diferentes modos de responder a la persona de Jesús y, a su vez, nos ayudará a entender a Nicodemo en su camino de discipulado, destacando dos elementos esenciales: la tensión constante del creer y la evolución gradual en el proceso de fe; estas reflexiones finalmente, nos invitan a realizar una confrontación personal respecto a nuestra propia búsqueda y encuentro con el Señor.

2 Significa "Vencedor del Pueblo", Martin menciona que existió un hombre, según escritos rabínicos, en tiempo de Jesús llamado Ben Gurion Naqdimon, que para algunos podría ser Nicodemo (MARTIN, 2002, p. 99).

3 El Sanedrín era ante todo el tribunal competente para decidir en última instancia sobre cuestiones relacionadas a la ley judía. 


\section{Nicodemo en la noche ${ }^{4}$ Juan 2, 23-3,21)}

El capítulo 2 del Evangelio de Juan inicia con el relato de las Bodas de Caná (2,12), donde Jesús realiza su primer signo y los discípulos creen en Él. Después el narrador ubica al lector en Jerusalén; allí se relata la expulsión de los vendedores del Templo (2,1322), al final del capítulo, el narrador introduce el tema de los signos y el seguimiento de la multitud a causa de ellos. Asimismo, terminando la perícopa, se presenta el personaje de Juan Bautista (Juan 3,22), lo que manifiesta claramente una nueva temática en el Evangelio y el inicio de otro relato. Así que, en este contexto narrativo, en medio de la expulsión de los vendedores del Templo y Juan Bautista, es presentado el personaje de Nicodemo.

El nombre de Nicodemo sólo aparece hasta el capítulo 3, versículo 1, pero hay razones para proponer que la perícopa inicia en el capítulo 2 , ya que en él se presenta la razón por la cual la gente sigue a Jesús: los signos; es esta la misma razón, que expondrá el narrador, para mostrar la motivación de Nicodemo para buscar a Jesús ${ }^{5}$.

El texto no nos presenta de manera directa el lugar, sólo sabemos que es Jerusalén; claramente es de noche y se puede concluir que es un lugar íntimo, tranquilo, donde pueden conversar con calma. Nicodemo busca a Jesús en la noche y sabe dónde encontrarlo, esto manifiesta ya un interés particular, sabemos que al inicio es un poco superficial, pues su primera afirmación hace referencia a los signos.

Al inicio del capítulo 3, Jesús y Nicodemo sostienen un diálogo sobre el nacer de nuevo. En el centro de esta temática se encuentra un malentendido y una ironía (v. 4), la polisemia joánica es clave en estas figuras. Nicodemo se ha detenido a pensar en el nacer y ha interpretado que Jesús habla de volver a nacer en el sentido humano pero el Maestro habla de nacer de lo alto, de nuevo en un sentido teológico. A Nicodemo le ha quedado claro qué significa nacer de nuevo, por eso del versículo 9 en adelante, el cómo ocupa el centro del relato. El camino es el creer ya no por lo signos.

Nicodemo desempeña el papel típico del "discípulo obtuso", habla en un nivel terrestre, "carnal", Jesús habla en un nivel celestial y espiritual; Nicodemo tiene el lenguaje de la vejez y la imposibilidad, en contraste, Jesús habla el idioma del nacimiento y la juventud. Esto permite al lector sopesar el discurso entre los hablantes (ISTITUTO TEOLOGICO MARCHIGIANO, 2021).

Comentario conclusivo: El relato se presenta como una historia, donde la revelación es progresiva ${ }^{6}$; se muestra a Jesús como un verdadero Maestro, y más adelante se revela su identidad (anagnorisis) como Hijo de Dios. Este Jesús debe ser aceptado o rechazado; Nicodemo todavía es tímido, será necesario que sea exaltado el Hijo de hombre para que aquellos que crean en Él, tengan vida eterna. En definitiva, el relato presenta al

4 La noche se ha interpretado de diversos modos, aquí nos interesa en un primer momento su contraste con la luz que se presenta al final del relato. Para Pietrantonio la expresión "vino de noche" no debe ser interpretada en un sentido estrictamente simbólica, pues para Nicodemo es claro que Jesús ha venido de parte de Dios (PIETRANTONIO, 2012, p. 67-84).

5 Esta posición la comparte Williford (1999), quien siguiendo el comentario de Harold Dodd afirma: "Hay varios puntos de conexión entre los versículos 23-25 y Juan 3,1-15. Uno de esos puntos es la mención de los signos, que dieron lugar a que muchos creyeran en el nombre de Jesús durante la semana de Pascua y la declaración de Nicodemo.”

6 Brown (2000, p. 179-180) afirma que el pensamiento joánico en esta perícopa es un proceso de conocimiento que tiene como referencia sucesiva a las tres personas divinas: el Espíritu (3-8), El Hijo (11-15) y el Padre (16-21). 
amor de Dios por el mundo que se manifiesta en la cercanía del Unigénito, con su presencia terrena y, sobre todo, en su exaltación en la cruz.

Nicodemo se acerca porque ha encontrado en Jesús algo que le causa un reconocimiento, todavía superficial, pero deja ver un hombre curioso, abierto a la escucha y que guarda silencio después de escuchar al Maestro. Vale la pena preguntarse ¿cuáles son las razones para buscar a Jesús en el hoy de nuestra historia? De hecho, la fe de Nicodemo en esta etapa es superficial porque es una fe en busca de signos, por lo que difícilmente podrá entender lo que el Maestro quiere enseñarle.

El relato deja ver a Nicodemo con la inquietud de trascendencia de eternidad y, aunque de algún modo, se está buscando a sí mismo, por lo menos se acerca al Maestro, quien le muestra un nuevo camino. Muchos, en algún momento de la vida se han acercado a Dios, igual que Nicodemo, por los signos o en busca de respuestas rápidas, sin embargo, en su infinita misericordia, el Señor usa estos primeros pasos para mostrar la ruta del camino que se debe recorrer: el camino de la fe, que también es un proceso en el cual se experimenta un cambio progresivo de la fe de los signos a la fe del encuentro.

\section{Nicodemo en medio del Sanedrín (Juan 7,45-53)}

El segundo texto se encuentra dentro de un relato amplio que presenta el capítulo 7, en el cual Jesús es rechazado por sus hermanos en Galilea; luego se hace presente en la fiesta de la Tiendas, allí se genera una confusión por su enseñanza y su origen, y tratan de capturarlo, pero no es posible; claramente, existe una división en la multitud: aquellos que lo aceptan y quienes lo rechazan. En el versículo 32 queda claro que los fariseos querían apresar a Jesús; entre los v.37 al 44, se contextualiza la narración afirmando que están en el día más importante de la fiesta de las Tiendas. El versículo 45 introduce una nueva escena en la que los guardias llegan donde los fariseos; finalmente, en el v. 53 se indica que todos regresan a sus casas.

En este relato, lo más posible es que Nicodemo se encuentre en el lugar donde se reunía el Sanedrín, lo que llama la atención es que están reunidos en el día más solemne de la fiesta, lo cual no era normal. Dicho lugar es conocido como Lishkat HaGazit ${ }^{7}$, el cual se encuentra sobre el muro occidental del Templo.

Se presenta una situación de división, de mucha tensión y, en medio de ella, está Nicodemo, como un fariseo (como uno de ellos), pero que toma la iniciativa al tratar de ser justo en un posible juicio; aunque se decide, su respuesta todavía es tímida. El narrador pone en boca de los fariseos la afirmación que ninguno de ellos cree en Jesús, e inmediatamente presenta a Nicodemo, como aquel que ya estuvo con el, a partir de una analepsis, recuerda lo ya acontecido.

Podríamos plantear que en este segundo texto nos encontramos con una complicación al acercarnos al personaje de Nicodemo, aún no hay una solución definitiva. El dilema de seguir a Jesús y creer en Él llega a un clímax donde Nicodemo trata de tomar partido, pero esto todavía no es suficiente. Al final del relato se afirma que cada uno va a su casa y Nicodemo permanece en silencio

7 Así llama la Mishná a la sede del Gran Sanedrín. Según Josefo este lugar es llamado boulh/, y estaba ubicado cerca del llamado Xystus, eso supone que estaba situado probablemente hacia el extremo occidental del mismo Monte del Templo (SCHÜRER, 1985, p. 507-524). 
Comentario conclusivo: Es claro que el narrador ha aumentado la tensión en torno a Jesús, la división y el deseo de capturarlo; el relato ambienta la situación que desembocará en la pasión y muerte del Señor. Nicodemo ya no busca a Jesús, sino que está con los suyos, ya no es de noche. Los fariseos afirman que ningún jefe le sigue y, en medio de esta afirmación el personaje levanta la voz, realmente él es un jefe que ha estado con el Maestro. Su raciocinio no es cuestionado, pues es el modo justo de proceder y los demás lo saben, más bien por el tono en el que es presentada la voz de Nicodemo, se le cuestiona que trate de ayudar a alguien de Galilea, y se le envía a estudiar. Aún no encontramos una solución definitiva para la trama de este personaje, la complicación es aún mayor, la trama ha tomado un nuevo giro por su iniciativa y aunque no es totalmente decidida, empieza a generar sospechas entre los suyos.

Es curioso que los jefes estén reunidos, pues no les estaba permitido hacer reuniones mientras se llevaba a cabo una celebración importante, como lo era la Fiesta de las Tiendas, puede verse cómo aquellos que tanto defendían la ley estaban quebrantando sus propios principios.

Entre el primero y el segundo relato se evidencia un cambio progresivo en la fe de Nicodemo; primero buscó a Jesús por los signos, pero ahora quiere escuchar al Maestro, no sólo verlo. Así que la curiosidad manifestada en la noche abrió las puertas al conocimiento; claramente, da el paso de lo accidental a lo esencial: escuchar. De alguna manera, ya no reconoce a Jesús solamente como Maestro sino como profeta. En este sentido, podríamos plantear varios interrogantes: ¿Quién es Jesús para nosotros hoy? ¿Existe el deseo ardiente de escuchar las palabras del profeta o sólo deseamos ver sus signos?

\section{Nicodemo en el sepulcro (Juan 19,38-42)}

El capítulo 19 del Evangelio de Juan nos presenta la pasión y muerte de Jesús. Los versículos 31-37 narran precisamente su muerte, y el versículo 38 inicia el relato de la sepultura. El capítulo concluye diciendo que dejaron en el sepulcro el cuerpo de Jesús. La pasión de Jesús es relatada en un modo solemne; el tema real está presente no sólo en el diálogo con Pilatos sino en el tono y los detalles presentados en la escena; esta indicación es importante para entender las acciones realizadas por Nicodemo en la narración.

Al inicio vemos a José de Arimatea pidiendo a Pilatos el cuerpo de Jesús; después de esto aparece Nicodemo, de quien se dice estuvo de noche con Jesús y trajo mirra y áloe, ambos envuelven el cuerpo de Jesús y lo ponen en el sepulcro. Ninguno de los personajes de este relato dice nada, las acciones son contadas directamente por el narrador, el sepulcro es un lugar de silencio en el Evangelio de Juan, el último que ha hablado de modo directo es Jesús al pronunciar sus últimas palabras: "Todo está cumplido".

Este relato nos presenta la solución definitiva frente al personaje de Nicodemo. De aquel fariseo que se presentó de noche, ya sólo se dice su nombre, no aparece su pertenencia a aquel grupo que se ha resistido a Jesús, más bien se puede asimilar al grupo que representa José de Arimatea y los discípulos escondidos; la noche aparece como una inclusión, para recordarnos quien era el personaje, pero ahora aparece a plena luz del día, realizando una acción de reconocimiento y respeto hacia el Maestro; nadie acude a él, nadie lo llama, es por su iniciativa que llega al lugar, portando una cantidad increíble de 
mirra y áloe. ${ }^{8}$ La mirra se usaba en Egipto para embalsamar cadáveres, los judíos la usaban para evitar el hedor de la putrefacción, es un aroma costoso; por otro lado el áloe es una madera aromática que servía para perfumar el lecho nupcial (Prov. 7,17) o las vestiduras reales (Sal 45,9). Este signo manifiesta el reconocimiento de Jesús como Rey; en consecuencia, podemos concluir, que las acciones demuestran un compromiso más serio en el camino de la fe, progresivamente se ve un discípulo más comprometido en el seguimiento de su Maestro.

El narrador presenta la escena de un modo rápido, no hay tiempo que perder, ya llega la Pascua, pero a pesar de la velocidad con que ocurren los hechos, se dan detalles interesantes, que son contados por el narrador con una intención teológica; la sepultura de Jesús se muestra desde una perspectiva real como lo ha hecho durante toda la pasión, no es sepultado como cualquiera, sino como un verdadero Rey.

Comentario conclusivo: El tercer relato ocurre en la Pascua, por tanto, es el momento del paso, del reconocimiento. Nicodemo trata a Jesús como el Rey, ha dado un paso más hacia el encuentro, trata el cuerpo de Jesús con solemne cuidado, derrama sobre Él mirra, dándole todo el respeto y la admiración. En este relato prima la acción sobre las palabras, ahora Nicodemo es un verdadero discípulo, que ha llegado a la fe del encuentro, al conocimiento pleno de quién es Jesús en su existencia, ha descubierto que Jesús es el Rey del universo.

Es el momento de contemplar a Jesús en la escena del sepulcro, de rendirle adoración, y de reconocer con enorme agradecimiento su gran sacrificio de amor; Es hora de preguntarse delante de Él ¿Hemos dado el paso al encuentro con Jesús Rey? ¿Nuestras acciones son coherentes con las de un verdadero discípulo?

\section{Consideraciones finales: valoración del personaje}

La figura de Nicodemo en el Evangelio de Juan es muy importante; su presencia en momentos claves del Evangelio así lo confirma. Desde la primera narración, en el diálogo, se resume el enfoque teológico de todo el cuarto Evangelio. La trama del personaje debe ser vista en todo su conjunto; los dos primeros episodios presentan dos complicaciones serias que terminan con el silencio del personaje, éstas solo encontrarán respuesta en el episodio final donde se recuerda quién era Nicodemo al inicio, el que llegó de noche, pero actúa de otro modo, la solución por fin ha llegado, aquel fariseo obra de una manera distinta, como un creyente.

La respuesta de Nicodemo a Jesús es progresiva, al principio es limitada temerosa y no comprende las cosas al plano de Jesús, aunque se reconoce que hay una búsqueda interior de este fariseo; los signos, la curiosidad y las preguntas, manifiestan el acercamiento inicial. En el segundo relato hay una evolución, se encuentra en otra condición, ahora se presenta ejerciendo su liderazgo en medio del pueblo y desde allí quiere escuchar a Jesús, buscando que se obre con justicia y honestidad, invita a otros a hacerlo, sin embargo, esta segunda escena está cargada de respetos humanos; aunque vemos iniciativa aun es tímido y temeroso. Al final las acciones hablan por él, el tercer relato lo muestra como un hombre nuevo; el respeto hacia Jesús y el modo de realizar su sepultura, muestran

8 Cien litros de mirra y aloe son 32 kilos y 70 gramos, cien veces la cantidad de perfume que María derramo sobre los pies de Jesús. 
a Jesús como un Rey, y es Nicodemo junto con José de Arimatea los encargados de presentar este sentido real al final del Evangelio.

En estos tres microrrelatos se presentan elementos que dejan ver los diferentes modos de responder a la persona de Jesús: de modo superficial, en contra de Él, o con una adhesión comprometida en acciones concretas. El tema del creer está presente en cada perícopa. Así pues, el tema de la fe es muy importante para el narrador, primero muestra todo aquello que se debe hacer para creer y qué se debe creer, después se muestra la necesidad de una respuesta en el camino de la fe; no se puede ser indiferente, y por último la existencia de un compromiso que supere al miedo o la timidez. Nicodemo aún no responde del modo justo en los dos primeros relatos; sólo al final se muestra una fe activa, no hay necesidad de las palabras. Jesús es su Rey.

Nicodemo es una persona importante para la sociedad judía, a Jesús no sólo lo sigue la gente humilde, sino que existe un interés en todos los niveles sociales, su presencia no pasa desapercibida para nadie. La voz del narrador nos ha presentado a Nicodemo en una constante tensión, donde se confronta la tradición judía y la novedad del anuncio Evangélico.

Nicodemo sirve al narrador para presentar un proceso de discipulado; la verdadera respuesta a Jesús en el Evangelio de Juan no consiste en palabras sino en acciones. El silencio de Nicodemo para algunos autores puede interpretarse de modo negativo pero el narrador nos permite ver a lo largo de estos silencios un cambio gradual. Cambio que permite concluir con una valoración positiva del personaje en el Evangelio de Juan, pues a pesar de su ambigüedad, timidez y temores, al final sus acciones son claras, su decisión incluso es mucho más coherente que la de los apóstoles. El discipulado pues, es presentado desde Nicodemo como un proceso dinámico que solo puede ser asumido en el caminar constante de la confrontación de la vida con el Maestro.

En Latinoamérica el primer anuncio que procura el encuentro con Jesús es una fortaleza, pero el proceso de discipulado es más discreto, hay sed de ver al maestro pero cuesta seguir sus pasos, Nicodemo dio el primer paso pero entendió en la confrontación de su vida que era necesario ir más allá, es hora no solo de ver y encontrarse con el Maestro es la hora de seguirlo en la cotidianidad de la existencia entregando la vida.

\section{Referencias}

BROWN, R. E. El Evangelio Según Juan I-XII. Madrid: Ediciones Cristiandad, 2000. v. 2.

CULPEPPER, R. A. Anatomy of the Fourth Gospel: A Study of the Literary Design. Philadelphia: Fortress Press, 1983.

ISTITUTO TEOLOGICO MARCHIGIANO. L 'incontro con Nicodemo: (Gv 3,1-21). Fermo: [s.n.]. Disponible en: http://www.teologiafermo.it/it/776/. Acceso en: 10 jun. 2021.

MARGUERAT, D.; BOURQUIN, Y. Per leggere i racconti biblici. Roma: Borla, 2011.

MARTIN, J. M. Personajes del Cuarto Evangelio. Madrid: Universidad Pontifica Comillas, 2002.

PIETRANTONIO, R. ¿Desde la concepción o por el nacimiento?: la conversación con Nicodemo (Juan 3, 1:21). Cuadernos de Teología, Buenos Aires, v. 21, p. 67-84, 2012.

SCHÜRER, E. Historia del pueblo judío en tiempos de Jesús 175 a.C.-135 d.C. Madrid: Ediciones Cristiandad, 1985. v. 2. 
WILLIFORD, Don. John 3,1-15-gennêthênai anôthen: A Radical Departure, A New Beginning. Review \& Expositor, Thousand Oaks, v. 96, n. 3, p. 451-461, 1999. Disponible en: https://journals.sagepub.com/doi/pdf/10.1177/003463739909600309. Acceso en: 10 jun. 2021.

ZAPELLA, L. Manuale di analisi narrativa bíblica. Torino: Claudiana, 2014. 